\title{
Heart rate variability analysis in acute poisoning by cholinesterase inhibitors
}

\author{
YONGIN KIM ${ }^{1}$, JINWOO JEONG ${ }^{2}$ \\ ${ }^{1}$ Department of Emergency Medicine, Pusan National University Yangsan Hospital \\ ${ }^{2}$ Department of Emergency Medicine, College of Medicine, Dong-A University \\ Corresponding author \\ Jinwoo Jeong \\ Department of Emergency Medicine \\ Dong-A University Hospital \\ Daesin Gongwon-Ro 26, Seogu, Busan, 49201 Republic of Korea \\ Phone: +82-51-240-2778 \\ Fax: +82-51-240-5309 \\ E-mail:advanced@lifesupport.pe.kr
}

\section{ABSTRACT}

Heart rate variability (HRV) has been associated with a variety of clinical situations. However, few studies have examined the association between HRV and acute poisoning. Organophosphate (OP) and carbamate inhibit esterase enzymes, particularly acetylcholinesterase, resulting in an accumulation of acetylcholine and thereby promoting excessive activation of corresponding receptors. Because diagnosis and treatment of OP and carbamate poisoning greatly depend on the severity of cholinergic symptoms, and because HRV reflects autonomic status, some HRV parameters may be of value in diagnosing OP and carbamate poisoning among patients visiting the emergency department.

Patients who visited the emergency department of the study hospital between September 2008 and May 2010 with the chief complaint of acute poisoning or overdose were included. Cases that involved ingestion of OP or carbamate insecticides were classified as poisoning by cholinesterase inhibitors and compared with other cases of poisoning or overdose. The timedomain analysis included descriptive statistics of R-R intervals and instantaneous heart rates. The frequency-domain analysis used fast Fourier transformation. A Poincaré plot, which is a scatterplot of R-R intervals against the preceding $\mathrm{R}-\mathrm{R}$ interval, was used for the nonlinear analysis.

Very-low-frequency (VLF) power and the ratio of low-frequency-to-high-frequency power (LF/HF) were the most effective parameters for distinguishing cholinesterase inhibitor poisoning among cases of acute poisoning, with areas under the receiveroperating characteristic curve of 0.76 and 0.87 , respectively. Cholinesterase inhibitor poisoning was a significant factor determining VLF power and the LF/HF ratio after adjusting for possible confounding variables, including age over 40, gender, and tracheal intubation.

Frequency-domain parameters of HRV, such as VLF power and the LF/HF ratio, might be considered as potential diagnostic methods to distinguish cholinesterase inhibitor poisoning from other cases of intoxication in the early stages of emergency care.

Key words: electrocardiography, organophosphates, carbamates, poisoning

\section{INTRODUCTION}

Heart rate dynamically responds to physiologic alterations, mediated by the autonomic nervous system via vagal and sympathetic nerve impulses. (1) Analysis of heart rate variability (HRV) using electrocardiographic (ECG) recording is an important method used to assess cardiovascular autonomic regulation. HRV has been associated with a variety of clinical situations such as mortality due to acute myocardial infarction, severity of trauma, diabetic neuropathy, outcome of prehospital ambulance patients, etc. (1-4) However, little research has examined the association between HRV and acute poisoning, except for antidepressant overdoses. $(5,6)$ Many parameters used to quantify HRV have been introduced and are categorized into time domain, frequency domain, and nonlinear parameters. Table 1 summarizes HRV parameters with a brief description of each. Time domain parameters are the most simple to analyze and are derived from statistical representation of R-R in- tervals or differences in consecutive R-R intervals. Frequency domain parameters are derived from power spectral density analysis and provide information on how power (variance) distributes as a function of frequency. $(1,4)$ It was reported that when parasympathetic activity is blocked, both LF and HF (low-frequency, high-frequency) values decrease, while LF power decreases by sympathetic blockade. (7) However, the effects of pharmacologic overstimulation of autonomic systems are not well-known. The Poincaré plot is one of several nonlinear methods used to analyze HRV, which is a scatterplot of the current $\mathrm{R}-\mathrm{R}$ interval plotted against the preceding R-R interval. The Poincaré plot is thought to be able to identify beat-to-beat patterns in data that are difficult to identify using frequency-domain analysis and can be quantified by standardized deviations along and perpendicular to the line of identity. (8)

Organophosphate (OP) and carbamate agents are widely used as pesticides in Asia. These compounds inhibit esterase enzymes, particularly acetylcholinesterase in synapses and on red cell membranes and butyrylcholinesterase in plasma. $(9,10)$ Acetylcholinesterase inhibition results in an accumulation of acetylcholine and thereby promotes excessive activation of corresponding receptors in synapses of the autonomic nervous system, central nervous system (CNS), and neuromuscular junctions. (9) Symptoms of OP and carbamate poisoning include cholinergic crisis, CNS dysfunction, and neuromuscular paralysis. $(9,11)$ Because diagnosis and treatment of OP and carbamate poisoning greatly depend on the severity of cholinergic symptoms and because HRV reflects autonomic status, the authors hy- 
pothesized that some HRV parameters may be of value in diagnosing OP and carbamate poisoning among patients visiting the emergency department (ED); thus, we conducted this study to evaluate the utility of HRV.

\section{MATERIALS AND METHODS}

This study was approved by the Institutional Review Board of the Pusan National University Hospital, and the need for written informed consent was waived by the Institutional Review Board because of the noninvasiveness of the study and impracticality of obtaining written consent in emergency settings. Patients who visited the emergency department of the study hospital between September 2008 and May 2010 with the chief complaint of acute poisoning or overdose were included. The ingested substances were identified by the patients or accompanying guardians if the patients were not communicable. Cases of pesticide poisoning were included only when the containers were identified. Cases that involved ingestion of organophosphate or carbamate insecticides were considered to be poisoning by cholinesterase inhibitors and were compared with other cases of poisoning or overdose.

A custom-built data-sampling device was connected to the analog ECG output port of a Lifepak 20 monitor-defibrillator (Physio-control, Redmond, WA). The device sampled the ECG signal at a rate of $1,000 \mathrm{~Hz}$ and transmitted the voltage data to a personal computer in numbers via Bluetooth wireless connection. On the personal computer, signal-receiving and -converting software that had been programmed by the authors stored the data in the form of a text file. The text file with the raw signal data was imported to the Physio Toolkit, (12) and R-R intervals were extracted from the ECG tracings. The quality of the ECG tracings and the validity of R-R interval extraction were manually verified by the author. Data with unsuitable quality to accurately determine $\mathrm{R}$ wave peaks due to artifacts and cases with atrial fibrillation were excluded from further analysis. The R-R intervals used for analyses were acquired from a 5-min period representing the earliest period of the patients' stay in the emergency department that provided data of appropriate quality. Emergency treatments, including tracheal intubation, intravenous access, and first dose of an antidote such as atropine, were given prior to signal acquisition. The HRV parameters used for analyses are summa- rized in table 1 . The time-domain analysis included descriptive statistics of R-R intervals and instantaneous heart rates. The frequency-domain analysis utilized fast Fourier transformation. Power spectral density against frequency is presented as a nonparametric Welch's periodogram. A Poincaré plot, which is a scatterplot of R-R intervals against the preceding R-R interval, was used for the nonlinear analysis of beat-to-beat patterns.

Gender, age, type and amount of ingested poisons, and prior treatments were extracted from medical records. Continuous variables with a normal distribution are presented as means and standard deviation (SD) and variables that do not conform to normal distribution are presented as median values with interquartile range. We compared data from cases of cholinesterase poisoning with other poisonings, because HRV parameters are sensitive to stress conditions, and standard normal and abnormal values cannot be defined, since the dynamic equilibrium of autonomic balance and its range of excursion can be extremely wide. (13) Categorical variables were compared using the chi-square test. Numerical variables were evaluated for normal distribution with the Shapiro-Wilk test, and thereafter a t-test or Mann-Whitney test were used for comparison, as appropriate. Receiver operating characteristic (ROC) curves were used to evaluate the diagnostic utility of HRV parameters for the diagnosis of cholinesterase inhibitor poisoning. The ROC curve is defined as a plot of test sensitivity as the $y$ coordinate versus its 1-specificity as the $x$ coordinate and is an effective method of evaluating the performance of diagnostic tests. $(14,15)$ Logistic regression analysis was used to determine the effects of possible confounding variables. The dependent variables were categorized as whether the HRV parameter of concern was above or below the proposed cutoff value. The independent variables included age over 40 , gender, whether the patient had altered consciousness or was intubated prior to HRV analysis, and whether the ingested toxin was a cholinesterase inhibitor.

HRV Analysis Software 1.1 (Biomedical Signal Analysis Group, Department of Applied Physics, University of Kuopio, Finland), (16) was used for the HRV analysis. MedCalc software (ver. 15.6; MedCalc Software, Mariakerke, Belgium) was used for the statistical analyses. Statistical significance was accepted when the p-value $<0.05$.

\section{RESULTS}

During the study period, a total of 110 cases were considered for inclusion. Among these, 27 cases were excluded because of poor signal quality, atrial fibrillation, and unknown toxins (figure 1).

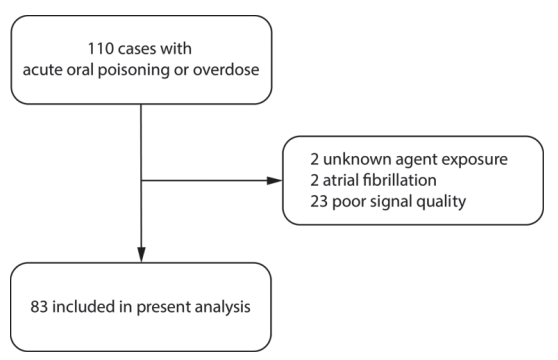

Figure 1. Flowchart of patient inclusion.

In total, 83 patients were included in the analyses; 45 (54.2\%) were males, and 38 $(45.8 \%)$ were females. The mean age of the patients was 48.5 (SD: 17.0) years. Among the 83 patients, eight had ingested cholinesterase inhibitors, and the other 75 had ingested various other substances. None of the eight patients with cholinesterase inhibitor poisoning had co-ingested other toxic materials. The ingested poisons are listed in table 2. Baseline characteristics and HRV parameters are summarized in table 3. Gender and age were not significantly different between the two groups. However, consciousness measured with AVPU (alert, verbal, pain, unresponsive) scale was worse in the cases with cholinesterase inhibitor poisoning, because cholinesterase inhibitors act as central nerve system depressants $(\mathrm{p}=0.013)$. All patients intoxicated with cholinesterase inhibitors received tracheal intubation, while only $36 \%$ of the other poisoned patients were intubated, $(p=0.001)$, which indicates the severe toxicity of cholinesterase inhibitors. Among the HRV parameters, only Verylow-frequency (VLF) power and the LF/ $\mathrm{HF}$ ratio had significance and were lower in the patients poisoned by cholinesterase inhibitors (figure 2).

The clinical and HRV characteristics of overdoses with common occurrence are listed in table 4 . HRV parameters did not differ much between groups, although statistical comparison was not possible because of substantial co-ingestions between these drugs. Poincaré plot samples are displayed in figure 3 for visual reference, although statistical significance was not found.

The ROC curve analyses were conducted to determine the diagnostic value of VLF power and the LF/HF ratio, which ap- 

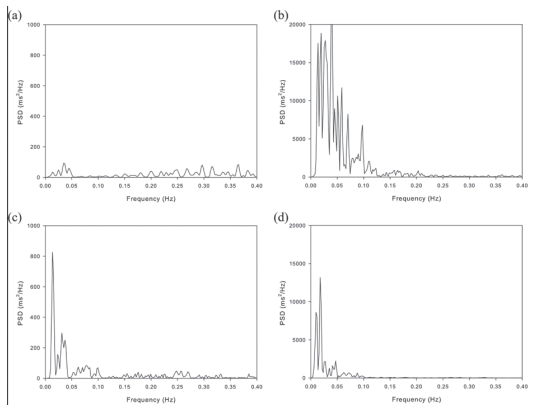

Figure 2. Welch's periodogram representing the frequency-domain analysis of a case of poisoning by (a) a cholinesterase inhibitor, (b) glyphosate herbicide, (c) benzodiazepine, and (d) zolpidem. The power spectrum patterns from cases of cholinesterase poisoning show decreased very-lowfrequency and low-frequency power. PSD, power spectrum density.

peared to be effective parameters, with areas under the ROC curve (AUC) values of 0.76 (95\% confidence interval [CI], $0.65-0.85 ; \mathrm{p}=0.045)$ and 0.87 (95\% CI, $0.78-0.94 ; \mathrm{p}<0.001$ ), respectively (figure $2)$. When a criterion of $<1.14 \mathrm{~ms} 2$ was used for VLF power, the sensitivity and specificity were $75.0 \%$ (95\% CI, 34.9-96.8) and $89.3 \%$ (95\% CI, 80.1-95.3), respective-

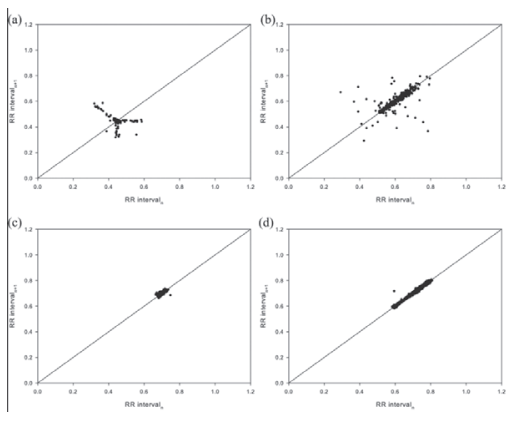

Figure 3. Poincaré plot of a case of poisoning by (a) a cholinesterase inhibitor, (b) glyphosate herbicide, (c) benzodiazepine, and (d) zolpidem. The plots are presented for visual reference. Quantized parameters were not statistically significant.

ly. The optimal cutoff value for the LF/HF ratio was 0.82 with a sensitivity of $100.0 \%$ (95\% CI, 63.1-100.0) and specificity of 69.3\% (95\% CI, 57.6-79.5).

The results of the logistic regression analyses are summarized in table 5 and table 6 . Cholinesterase inhibitor poisoning was a significant factor determining VLF power and the LF/HF ratio after adjusting for
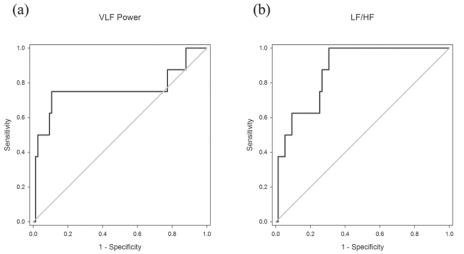

Figure 4. Receiver-operating characteristic curves of (a) very-low-frequency power and (b) the low-frequency-to-high-frequency power ratio for the diagnosis of poisoning by cholinesterase inhibitors.

Table 1. List of HRV parameters used in the analyses $(2,4,8,26)$.

\begin{tabular}{ll}
\hline Parameter & Description \\
\hline Time-domain analysis & Standard deviation of R-R intervals \\
\hline SDNN (s) & Root of mean squared differences between adjacent R-R intervals \\
\hline RMSSD (s) & $\begin{array}{l}\text { Number of interval differences greater than } 50 \text { ms in consecutive R-R } \\
\text { intervals }\end{array}$ \\
\hline pNN50 (\%) & Proportion of NN50 to total number of R-R intervals \\
\hline Frequency-domain analysis & \\
\hline VLF (ms2) & Power in the very-low-frequency range $(\leq 0.04 \mathrm{~Hz})$ \\
\hline LF (ms2) & Power in the low-frequency range $(0.04-0.15 \mathrm{~Hz})$ \\
\hline HF (ms2) & Power in the high-frequency range $(0.15-0.40 \mathrm{~Hz})$ \\
\hline LF/HF & Ratio of LF-to-HF \\
\hline Poincaré plot analysis & \\
\hline Width & Standard deviation of points perpendicular to the line of identity \\
\hline Length & Standard deviation of points along the line of identity \\
\hline Length/width & Proportion of length to width \\
\hline
\end{tabular}

HRV, heart rate variability.

Values are presented as means \pm standard deviation 
Table 2. Ingested substances and number of exposures.

\begin{tabular}{|c|c|}
\hline Substance ingested & Number of exposures (\%) \\
\hline \multicolumn{2}{|l|}{ Cholinesterase inhibitors } \\
\hline Organophosphate insecticides & $5(6.0)$ \\
\hline Carbamate insecticides & $3(3.6)$ \\
\hline \multicolumn{2}{|c|}{ Other ingested substances that caused poisoning or overdose } \\
\hline Tetrodotoxin & $4(4.8)$ \\
\hline Cyanide & $1(1.2)$ \\
\hline Acetaminophen & $6(7.2)$ \\
\hline Non-steroidal anti-inflammatory drugs & $4(4.8)$ \\
\hline Beta-adrenergic blockers & $3(3.6)$ \\
\hline Calcium-channel blockers & $1(1.2)$ \\
\hline Haloperidol & $1(1.2)$ \\
\hline Amphetamine & $1(1.2)$ \\
\hline Benzodiazepines & $20(24.1)$ \\
\hline Zolpidem & $12(14.5)$ \\
\hline Seroquel & $3(3.6)$ \\
\hline Antihistamines & $8(9.6)$ \\
\hline Cyclic antidepressants & $3(3.6)$ \\
\hline Selective serotonin reuptake inhibitors & $11(13.3)$ \\
\hline Serotonin antagonist and reuptake inhibitors & $3(3.6)$ \\
\hline Serotonin-norepinephrine reuptake inhibitors & $1(1.2)$ \\
\hline Benztropine & $1(1.2)$ \\
\hline Perphenazine & $2(2.4)$ \\
\hline Pyrethroids & $4(4.8)$ \\
\hline Paraquat & $2(2.4)$ \\
\hline Glyphosate & $9(10.8)$ \\
\hline Glufosinate & $2(2.4)$ \\
\hline Benzoylurea insecticide & $1(1.2)$ \\
\hline Methoxy insecticide & $1(1.2)$ \\
\hline Benzoate herbicide & $1(1.2)$ \\
\hline Indoxacarb & $1(1.2)$ \\
\hline Ephedrine & $1(1.2)$ \\
\hline Caffeine & $1(1.2)$ \\
\hline Valproate & $1(1.2)$ \\
\hline
\end{tabular}

Note: The numbers do not add up to the total number of patients (83) because some patients ingested more than one substance.

Table 3. Baseline characteristics and heart rate variability parameters of patients with cholinesterase inhibitor poisoning and other poisonings. Categorical variables are presented as the frequency and percentage. Numerical variables are presented as means \pm standard deviation or as median values (interquartile range), as appropriate.

\begin{tabular}{llll}
\hline & $\begin{array}{l}\text { Cholinesterase inhibitor poison- } \\
\text { ing }(\mathbf{n}=\mathbf{8})\end{array}$ & $\begin{array}{l}\text { Other poisoning } \\
(\mathbf{n}=75)\end{array}$ & NS $^{*}$ \\
\hline Gender & & & \\
\hline Male & $6(75.0)$ & $39(52.0)$ & $\mathrm{NS}^{* *}$ \\
\hline Female & $2(25.0)$ & $36(48.0)$ & $0.013^{*}$ \\
\hline Age (years) & $59.5 \pm 11.6$ & $48.1 \pm 16.7$ & \\
\hline Consciousness & & $34(45.3)$ & \\
\hline Alertness & $1(12.5)$ & $18(24.0)$ & \\
\hline Response to verbal stimuli & $2(25.0)$ & &
\end{tabular}




\begin{tabular}{|c|c|c|c|}
\hline Response to pain & $2(25.0)$ & $19(25.3)$ & \\
\hline Unresponsive & $2(37.5)$ & $4(5.3)$ & \\
\hline Tracheal intubation & $8(100.0)$ & $27(36.0)$ & $0.001^{*}$ \\
\hline \multicolumn{4}{|c|}{ Time-domain analysis } \\
\hline SDNN (s) & $0.03(0.01-0.04)$ & $0.02(0.01-0.03)$ & $\mathrm{NS}^{* *}$ \\
\hline RMSSD (s) & $34.28(9.86-54.71)$ & $17.58(9.15-29.32)$ & $\mathrm{NS}^{* *}$ \\
\hline NN50 (count) & $13(4.50-44.50)$ & $3(1.0-10.0)$ & $\mathrm{NS}^{* *}$ \\
\hline pNN50 (\%) & $2.12(0.72-7.51)$ & $0.81(0.18-2.22)$ & $\mathrm{NS}^{* *}$ \\
\hline \multicolumn{4}{|c|}{ Frequency-domain analysis } \\
\hline VLF (ms2) & $0.67(0.20-49.87)$ & $24.69(4.71-77.95)$ & $0.016^{* * *}$ \\
\hline $\mathrm{LF}(\mathrm{ms} 2)$ & $1.26(0.69-252.70)$ & $21.31(2.53-67.97)$ & $\mathrm{NS}^{* * *}$ \\
\hline $\mathrm{HF}(\mathrm{ms} 2)$ & $11.29(3.43-332.22)$ & $11.38(1.95-46.15)$ & $\mathrm{NS}^{* * *}$ \\
\hline $\mathrm{LF} / \mathrm{HF}$ & $0.24(0.10-0.68)$ & $1.39(0.58-3.85)$ & $0.001^{\star * *}$ \\
\hline \multicolumn{4}{|l|}{ Poincaré plot analysis } \\
\hline Width & $28.48(9.75-43.01)$ & $14.73(6.97-23.63)$ & $N S^{* * *}$ \\
\hline Length & $25.71(9.237-45.612)$ & $34.46(19.31-59.00)$ & $\mathrm{NS}^{* * *}$ \\
\hline Length/width & $1.24(0.73-2.11)$ & $1.77(0.84-3.67)$ & NS \\
\hline
\end{tabular}

Abbreviations are the same as in table 1.

* Compared with chi-square test

** Compared with independent sample t-test

*** Compared with Mann-Whitney test

Table 4. Clinical and heart rate variability parameters of overdoses of larger cases.

\begin{tabular}{|c|c|c|c|}
\hline & Benzodiazepines $(n=20)$ & Zolpidem $(n=12)$ & $\operatorname{SSRI}^{\star}(\mathrm{n}=11)$ \\
\hline \multicolumn{4}{|l|}{ Gender } \\
\hline Male & $8(40.0)$ & $2(16.7)$ & $4(36.4)$ \\
\hline Female & $12(60.0)$ & $10(83.3)$ & $7(63.6)$ \\
\hline Age (years) & $43.5 \pm 17.5$ & $44.1 \pm 19.2$ & $36.8 \pm 14.4$ \\
\hline \multicolumn{4}{|l|}{ Consciousness } \\
\hline Alertness & $6(30.0)$ & $4(33.3)$ & $3(27.3)$ \\
\hline Response to verbal stimuli & $8(40.0)$ & $3(25.0)$ & $4(36.4)$ \\
\hline Response to pain & $6(30.0)$ & $5(41.7)$ & $4(36.4)$ \\
\hline Unresponsive & $0(0.0)$ & $0(0.0)$ & $0(0.0)$ \\
\hline Tracheal intubation & $6(30.0)$ & $5(41.7)$ & $3(27.3)$ \\
\hline \multicolumn{4}{|l|}{ Time-domain analysis } \\
\hline $\operatorname{SDNN}(s)$ & $0.03(0.02-0.05)$ & $0.02(0.01-0.03)$ & $0.28(0.01-0.05)$ \\
\hline RMSSD (s) & $18.61(8.05-34.35)$ & $14.34(6.42-21.79)$ & $22.21(10.91-50.16)$ \\
\hline NN50 (count) & $2(0.00-8.50)$ & $3(0.00-9.50)$ & $2(1.00-15.00)$ \\
\hline pNN50 (\%) & $0.75(0.00-2.40)$ & $0.63(0.00-1.95)$ & $0.73(0.19-3.62)$ \\
\hline \multicolumn{4}{|l|}{ Frequency-domain analysis } \\
\hline VLF (ms2) & $35.92(14.21-148.76)$ & $53.01(7.22-201.85)$ & $24.06(15.03-168.73)$ \\
\hline $\mathrm{LF}(\mathrm{ms} 2)$ & $35.02(16.13-99.04)$ & $33.41(1.16-66.74)$ & $27.27(11.67-107.89)$ \\
\hline $\mathrm{HF}(\mathrm{ms} 2)$ & $18.70(4.08-63.37)$ & $5.87(0.83-42.59)$ & $11.38(4.03-94.71)$ \\
\hline $\mathrm{LF} / \mathrm{HF}$ & $1.25(0.83-5.37)$ & $1.23(0.63-7.08)$ & $1.08(0.88-3.24)$ \\
\hline \multicolumn{4}{|l|}{ Poincaré plot analysis } \\
\hline Width & $14.96(8.69-25.32)$ & $10.69(4.87-15.89)$ & $16.29(7.85-36.04)$ \\
\hline Length & $46.72(30.49-74.44)$ & $49.05(24.47-82.45)$ & $53.06(27.67-78.30)$ \\
\hline Length/width & $2.33(0.89-3.51)$ & $5.62(3.14-9.61)$ & $1.82(0.83-3.47)$ \\
\hline
\end{tabular}

Abbreviations are the same as in table $1 .{ }^{*}$ SSRI: selective serotonin reuptake inhibitor 
Table 5. Multivariate logistic regression models used to determine an association between cholinesterase inhibitor poisoning and very-low frequency power (VLF) below the proposed cutoff value (1.137 ms2) adjusted for gender, age over 40, alertness, and prior tracheal intubation. The total number of cases is $83 ; V L F<1.137$ ms 2 cases are $14 ; V L F \geq 1.137$ ms 2 cases are 69.

\begin{tabular}{lllll}
\hline Variable & Coefficient & S.E. & p-value & Odds ratio $\left(\mathbf{9 5 \%}\right.$ CI $\left.{ }^{* *}\right)$ \\
\hline $\begin{array}{l}\text { Cholinesterase inhibitor } \\
\text { poisoning }\end{array}$ & 2.13 & 0.96 & 0.027 & $8.40(1.28-55.15)$ \\
\hline Male gender & 0.11 & 0.75 & 0.887 & $1.11(0.26-4.83)$ \\
\hline Age over 40 & 1.24 & 1.14 & 0.280 & $3.44(0.37-32.30)$ \\
\hline Tracheal intubation & 1.81 & 0.87 & 0.037 & $6.14(1.12-33.60)$ \\
\hline Constant & -4.21 & 1.77 & 0.017 & Not applicable
\end{tabular}

Model chi-square $=22.56(\mathrm{p}<0.001)$

Nagelkerke R2 $=0.40$

Hosmer and Lemeshaw $\mathrm{p}=0.613$

${ }^{*}$ S.E.: standard error ${ }^{*} \mathrm{CI}$ : confidence interval

Table 6. Multivariate logistic regression models used to determine an association between cholinesterase inhibitor poisoning and the ratio of low-frequency power to high-frequency power ( $L F / H F)$ below the proposed cutoff value (0.82) adjusted for gender, age over 40, alertness, and prior tracheal intubation. The total number of cases was $83 ; \mathrm{LF} / \mathrm{HF}<0.82$ cases were $30 ; \mathrm{LH} / \mathrm{HF} \geq 0.82$ cases were 53 .

\begin{tabular}{lllll}
\hline Variable & Coefficient & S.E. & p-value & Odds ratio $\left(\mathbf{9 5 \%} \mathbf{C I}^{* *}\right)$ \\
\hline $\begin{array}{l}\text { Cholinesterase inhibitor } \\
\text { poisoning }\end{array}$ & 2.42 & 1.17 & 0.038 & $11.22(1.15-110.05)$ \\
\hline Male gender & 0.89 & 0.53 & 0.098 & $2.42(0.85-6.90)$ \\
\hline Age over 40 & -0.06 & 0.57 & 0.910 & $0.94(0.31-2.86)$ \\
\hline Tracheal intubation & 1.09 & 0.54 & 0.042 & $2.98(1.04-8.55)$ \\
\hline Constant & -2.55 & 1.05 & 0.015 & Not applicable \\
\hline
\end{tabular}

Model chi-square $=16.84(\mathrm{p}=0.002)$

Nagelkerke R2 $=0.25$

Hosmer and Lemeshaw $\mathrm{p}=0.995$

${ }^{\star}$ S.E.: standard error ${ }^{* *} \mathrm{CI}$ : confidence interval

\section{DISCUSSION}

HRV was first appreciated as a clinical tool for monitoring fetal distress in 1965. (4) Currently, the association of HRV and autonomic function is widely appreciated in clinical medicine. (1-4,13,17-21) As autonomic dysfunction largely characterizes the symptoms caused by cholinesterase inhibitor intoxication, HRV may be of diagnostic value in cases of pesticide poisoning. Our data showed that HRV parameters, particularly frequency-domain results of the VLF and LF/HF areas, were associated with organophosphate and carbamate poisoning.

This study demonstrated that the time-domain analyses were not significant. Timedomain results are usually indicators of gross variation in the R-R interval and are attributed to the respiratory cycle. $(1,2,4)$ To assess specific autonomic dysfunction, more sophisticated analyses, such as transformation to frequency domain, would be needed.

There is some debate regarding the interpretation of frequency-domain results, but it is generally believed that HF power reflects ventilator modulation of $\mathrm{R}-\mathrm{R}$ intervals, and LF power is modulated by both sympathetic and parasympathetic nerve traffic to the heart. $(1,4)$ Although LF power and HF power alone did not have significant diagnostic value based on the ROC curve, the LF/HF ratio exhibited good sensitivity and specificity. A possible explanation could be that the overwhelming activation of the autonomic nervous system caused by cholinesterase inhibitors suppressed LF power more than HF power. Cholinesterase inhibitors are known to exert unregulated and excessive stimulation of both muscarinic and nicotinic receptors in the central and autonomic nervous systems due to an accumulation of acetylcholine, and the symptoms are commonly referred to as 'acute cholinergic crisis'. $(11,22)$ Although the physiological basis for VLF power is far more complex than HF and LF power, with some authors recommending that VLF be used in the interpretation of short-term HRV data, (4) it has been suggested that VLF power is associated with parasympathetic activity. (1) In our study,
VLF was a significant predictor of poisoning by cholinesterase inhibitors. We speculate that VLF showed a promising result because cholinesterase inhibitors influence parasympathetic activity more potently than other clinical conditions studied previously.

The Poincaré plot is a nonlinear method for analyzing HRV data, which provides graphical understanding of beat-to-beat cycles and patterns not apparent in frequency-domain analyses. (8) It is a scatterplot of the each $\mathrm{R}-\mathrm{R}$ interval plotted against the preceding $\mathrm{R}-\mathrm{R}$ interval. The standard deviation of points perpendicular to the line of identity (the width) represents short-term variation and is correlated with the root mean square successive difference (RMSSD). The standard deviation of points along the line of identity (length) represents long-term variation and is reported to represent parasympathetic blockade caused by complete denervation or atropine use. Complete denervation caused by cardiac transplantation produced a Poincaré plot of very small length and width, while atropine infusion only reduced the width of 
the Poincaré plot, resulting in an increased length/width ratio. $(8,23)$ Our data show that the values derived from the Poincaré plot are not useful indicators of cholinesterase poisoning, and that the pattern itself is unlike previously reported atropine effects or denervation, which could possibly be explained by the fact that the autonomic disturbance caused by cholinesterase inhibitors represents an autonomic overload, unlike atropine-derived autonomic blockade.

A number of studies have indicated the usefulness of HRV analysis in both emergency and nonemergency settings, and some have even suggested that HRV could be regarded as a new vital sign. $(24,19,24,25)$ One of the major limitations of using HRV analysis in emergency situations is the complexity involved in processing and analyzing the HRV signal. (19) In this study, the time required to process HRV data from each case was largely dependent on the reliability of the automatic R-wave peak-detection algorithm. Some cases had ECG waveforms that were hard to process using automatic methods, and considerable effort was needed to manually verify and edit the R-R interval data in these cases. Development of devices with automatic frequency-domain analysis along with reliable peak-detection algorithms would be required prior to widespread adoption of HRV analysis in emergency settings.

This study was limited by the small number of subjects. Although the total number of cases analyzed was 83 , there were only 8 positive cases. Our results should be confirmed by a larger study. Also, dose relationships and HRV changes due to therapeutic measures should be studied. There are concerns that emotion and other factors also contribute to the HRV results and therefore the 'normal range' is very wide. $(1,4)$ The authors tried to minimize this confounding effect by comparing patients with others in the same emergency department setting rather than with previously reported normal ranges from healthy subjects.

Treatments, such as intravenous access or tracheal intubation, were given prior to HRV analysis. However, the limitation is unavoidable in an acute care setting, even for established diagnostic tests, where treatment takes priority over diagnosis. We tried to control for the confounding effect by utilizing multivariate logistic regression analysis.

Atropine is used to treat poisoning by cholinesterase inhibitors very early in the treatment course. Although atropine was used before the ECG signal was captured, our data reflect a very early condition before full atropinization was accomplished. A previous study revealed that parasympathetic blockade by atropine decreased LF and HF power, (7) while our results found VLF power and the LF/HF ratio useful for diagnosing cholinesterase inhibitor poisoning. The pattern appearing on the Poincaré plot from cases of cholinesterase poisoning was unlike the previously reported atropine effect. Thus, HRV analysis in this study explains the effect of cholinesterase poisoning more than that of atropine.

\section{CONCLUSION}

Frequency-domain analysis of heart rate variability showed significantly lower VLF power and LF/HF ratio in cases involving cholinesterase inhibitor poisoning compared to other cases of intoxication in the early stages of emergency care.

\section{ACKNOWLEDGEMENT}

This work was supported by the Dong-A University Research Fund.

\section{REFERENCES}

1. Kleiger RE, Stein PK, Bigger JT, Jr. Heart rate variability: measurement and clinical utility. Ann Noninvasive Electrocardiol 2005;10(1):88-101.

2. Ong ME, Padmanabhan P, Chan YH, Lin Z, Overton J, Ward KR, et al. An observational, prospective study exploring the use of heart rate variability as a predictor of clinical outcomes in pre-hospital ambulance patients. Resuscitation 2008;78(3):289-97.

3. Proctor KG, Atapattu SA, Duncan RC. Heart rate variability index in trauma patients. J Trauma 2007;63(1):33-43.

4. Task Force of the European Society of Cardiology and the North American Society of Pacing and Electrophysiology. Heart rate variability: standards of measurement, physiological interpretation and clinical use. Task Force of the European Society of Cardiology and the North American Society of Pacing and Electrophysiology. Circulation 1996;93(5):1043-65.

5. Dinleyici EC, Kilic Z, Sahin S, Tutuncu-Toker R, Eren M, Yargic ZA, et al. Heart rate variability in children with tricyclic antidepressant intoxication. Cardiol Res Pract 2013;2013:196506.

6. Waring WS, Rhee JY, Bateman DN, Leggett GE, Jamie H. Impaired heart rate variability and altered cardiac sympathovagal balance after antidepressant overdose. Eur J Clin Pharmacol 2008;64(11):1037-41.

7. Bolea J, Pueyo E, Laguna P, Bailon R. Non-linear HRV indices under autonomic nervous system blockade. Conf Proc IEEE Eng Med Biol Soc 2014;2014:3252-5.

8. Brennan M, Palaniswami M, Kamen P. Poincare plot interpretation using a physiological model of HRV based on a network of oscillators. Am J Physiol Heart Circ Physiol 2002;283(5):H1873-86.

9. Eddleston M, Buckley NA, Eyer P, Dawson AH. Management of acute organophosphorus pesticide poisoning. Lancet 2008;371(9612):597-607.

10. Rosman Y, Makarovsky I, Bentur Y, Shrot S, Dushnistky T, Krivoy A. Carbamate poisoning: treatment recommendations in the setting of a mass casualties event. Am J Emerg Med 2009;27(9):1117-24.

11. Iyer R, Iken B, Leon A. Developments in alternative treatments for organophosphate poisoning. Toxicol Lett 2015;233(2):200-6.

12. Goldberger AL, Amaral LA, Glass L, Hausdorff JM, Ivanov PC, Mark RG, et al. PhysioBank, PhysioToolkit, and PhysioNet: components of a new research resource for complex physiologic signals. Circulation 2000;101(23):E215-20.

13. Malliani A. Heart rate variability: from bench to bedside. Eur J Intern Med 2005;16(1):12-20.

14. Cicero MX, Walsh B, Solad Y, Whitfill T, Paesano G, Kim K, et al. Do you see what I see? Insights from using google glass for disaster telemedicine triage. Prehosp Disaster Med 2015;30(1):4-8.

15. Park SH, Goo JM, Jo CH. Receiver operating characteristic (ROC) curve: practical review for radiologists. Korean J Radiol 2004;5(1):11-8. 
16. Niskanen JP, Tarvainen MP, Ranta-Aho PO, Karjalainen PA. Software for advanced HRV analysis. Comput Methods Programs Biomed 2004;76(1):73-81.

17. Huikuri HV, Makikallio T, Airaksinen KE, Mitrani R, Castellanos A, Myerburg RJ. Measurement of heart rate variability: a clinical tool or a research toy? J Am Coll Cardiol 1999;34(7):1878-83.

18. Khandoker AH, Jelinek HF, Palaniswami M. Heart rate variability and complexity in people with diabetes associated cardiac autonomic neuropathy. Conf Proc IEEE Eng Med Biol Soc 2008;2008:4696-9.

19. King DR, Ogilvie MP, Pereira BM, Chang Y, Manning RJ, Conner JA, et al. Heart rate variability as a triage tool in patients with trauma during prehospital helicopter transport. J Trauma 2009;67(3):436-40.

20. Liu T, Lin Z, Ong ME, Koh ZX, Pek PP, Yeo YK, et al. Manifold ranking based scoring system with its application to cardiac arrest prediction: A retrospective study in emergency department patients. Comput Biol Med 2015;67:74-82.

21. Suzuki A, Yoshioka K, Ito S, Naito Y. Assessment of stress and autonomic nervous activity in Japanese female ambulance paramedics working 24-hour shifts. J Occup Health 2016;58:47-55.

22. Rosenbaum C, Bird SB. Non-muscarinic therapeutic targets for acute organophosphorus poisoning. J Med Toxicol 2010;6(4):408-12.

23. Esperer HD, Oehler M. Automatic quantification of the Poincare plot asymmetry of NN-interval recordings. Physiol Meas 2010;31(3):395-413.

24. Mohebbi M, Ghassemian H. Prediction of paroxysmal atrial fibrillation based on non-linear analysis and spectrum and bispectrum features of the heart rate variability signal. Comput Methods Programs Biomed. 2012;105(1):40-9.

25. Montano N, Porta A, Cogliati C, Costantino G, Tobaldini E, Casali KR, et al. Heart rate variability explored in the frequency domain: a tool to investigate the link between heart and behavior. Neurosci Biobehav Rev 2009;33(2):71-80.

26. Bigger JT, Jr., Fleiss JL, Steinman RC, Rolnitzky LM, Schneider WJ, Stein PK. RR variability in healthy, middle-aged persons compared with patients with chronic coronary heart disease or recent acute myocardial infarction. Circulation 1995;91(7):1936-43. 